\title{
Image-Based Planning and Validation of C1-C2 Transarticular Screw Fixation Using Personalized Drill Guides
}

\author{
Kirsten Martens ${ }^{1}$, Kris Verstreken ${ }^{1}$, Johan Van Cleynenbreugel ${ }^{1}$, \\ Karel Van Brussel ${ }^{3}$, Jan Goffin ${ }^{2}$, Guy Marchal ${ }^{1}$, and Paul Suetens ${ }^{1}$ \\ ${ }^{1}$ Laboratory for Medical Image Computing (ESAT and Radiology), K.U.Leuven \\ 2 Department of Neurosurgery \\ University Hospitals of Leuven, Herestraat 49, B-3000 Leuven, Belgium \\ 3 Department of Biomechanics and Engineering Design \\ Celestijnenlaan 200A, B-3001 Heverlee, Belgium \\ Kirsten.Martens@uz.kuleuven.ac.be
}

\begin{abstract}
Posterior transarticular spine fusion is a surgical procedure used to stabilize the cervical bodies $\mathrm{C} 1$ and $\mathrm{C} 2$. Currently, spine screws are used most frequently, according to the procedure of Magerl. As the anatomy is rather complex and the view is limited, this procedure has a high risk factor. Thus we present and validate a planning system for cervical screw insertion based on preoperative CT imaging. It allows a neurosurgeon to interactively determine the desired position of the cervical screws, based on appropriate and in real-time calculated reslices through the preoperative CT data. Guided by this information, a personalized mechanical drill guide is produced. The system is validated by five cadaver experiments. Based on the preoperative planning, screws are inserted through the drill guide. A semi-automatic technique is used to extract the screw locations from the postoperative images. The deviations of the axes of the planned and the inserted screws are determined in this way. We have observed that the drill guides are not yet stable enough to cope with the drilling forces that they are subjected to. As a result, they tend to displace. However, most of the inserted screws were judged to be adequately placed. No vascular compromise nor invasion of the spinal canal was observed.
\end{abstract}

Keywords Image-based surgery, C1-C2 instability, personalized drill guides

\section{Introduction}

Posterior transarticular screw fixation of the spinal segment C1-C2 has gained an increasing popularity. This technique was introduced by Magerl and Seemann [1] and has been widely used for atlantoaxial instability [2,3]. This instability poses special stabilization challenges compared with the remainder of the cervical spine. The unique architecture of the atlantoaxial complex (Figure 1a 
and $1 \mathrm{~b}$ ), with its horizontal articular surfaces, ligamentous connections and distinctive vertebral anatomy, allows not only flexion, extension and lateral bending, but also significant axial rotation [4]. C1-C2 transarticular screw fixation has become popular because it achieves greater biomechanical stability and superior fixation of atlantoaxial rotation [5] compared with the standard fusion techniques described by Gallie [6] or Brooks and Jenkins [7]. The success of this technique has been demonstrated in a number of clinical series $[2,3,5,9,10,11]$. However, despite of the reported success rates ranging from 87 to $100 \%$, the variable anatomy of the atlantoaxial complex often limits the use of the transarticular screw technique. The technique is potentially dangerous because the screw path is close to important structures. The following complications associated with this procedure have been reported: malpositioning of screws, pseudoarthrosis, implant failure, dural tear, neurologic deficit and vascular compromise causing brain stem infarction and death $[12,3,13]$. Consequences of injuries of the vertebral artery range from occult arteriovenous fistula to frank arterial compromise $[12,14]$. When inserting the screw the surgeon uses his or her anatomic knowledge to align the drill in the proper direction. A significant error may be induced by only a slight deviation of the direction. To check the correct placement of the screw in the pedicle, fluoroscopy is used. However, the vertebral artery, which is particularly at risk at the isthmus of $\mathrm{C} 2$, can not be visualized, neither directly, nor by fluoroscopy.

In order to enhance the surgeon's confidence in placing the screws, we have developed a novel technique that combines preoperative computer tomography with the use of a personalized mechanical drill guide produced by stereolithography. After a description of the system and the method, a first validation on five cadavers is presented. Quantitative and qualitative results are shown. At the time of writing 3 more cadavers and 2 real patients are in the planning stage.

\section{Surgery Planning and Support}

\subsection{About the Planning System and Steps}

The planning system for endosseous oral implants [15] has been adapted for planning the insertion of $\mathrm{C} 1-\mathrm{C} 2$ transarticular screws. In short, the user is working in a virtual environment wherein all objects are located. This scenery is viewed by multiple cameras from random viewing positions and with various viewing angles and zoom factors [15]. All objects present can be manipulated by the user to suit his or her viewing needs. The major modification made to the existing system was the incorporation of the possibility to make $2 \mathrm{D}$ reslices perpendicular to and containing the inserted screws. This is an indispensable feature for assessing the correct placement of the screws in 3 dimensions, especially within the joint itself.

A stepwise account of the planning procedure is now given. Insertion of the virtual screw is done as follows. A screw, represented as a cylinder with a central axis, is defined using two points named origin and end point. The cylinder has a transparent safety zone around it that indicates a distance of $2 \mathrm{~mm}$. Using the 


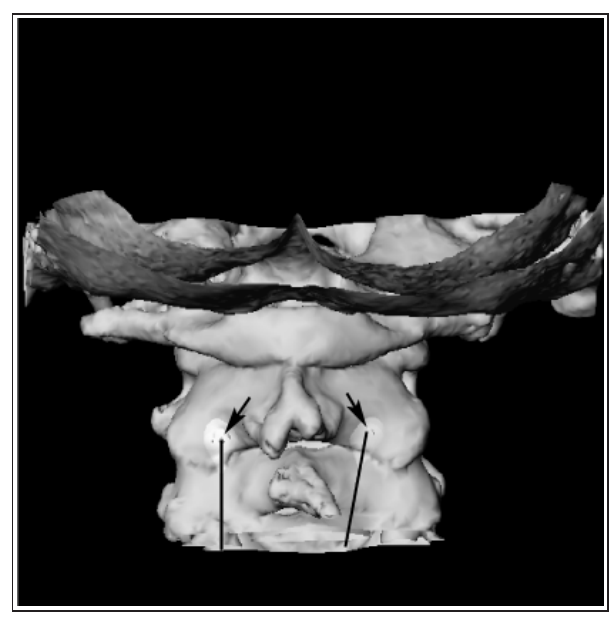

(a)

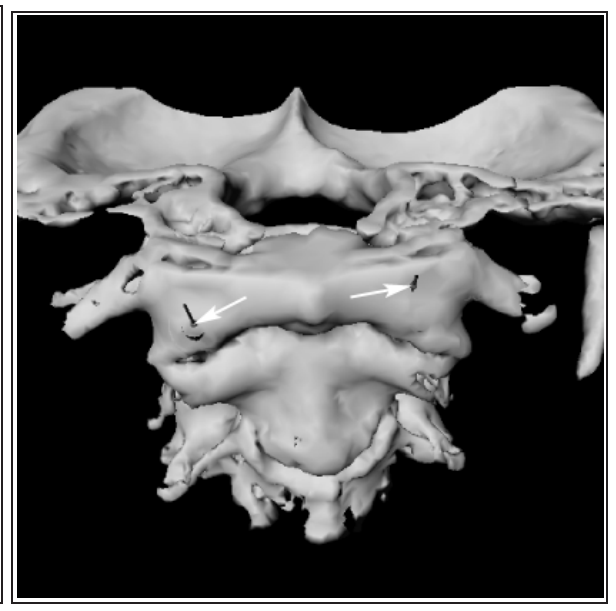

(b)

Fig. 1. (a) Full posterior view of the C1-C2 complex and skull base, with arrows indicating the entry points (origins) of the transarticular screws at C2 (b) Full anterior view of the $\mathrm{C} 1-\mathrm{C} 2$ complex and skull base, with arrows indicating the exit points (end points) of the transarticular screws at the anterior cortical bone of $\mathrm{C} 1$

CT-derived surface model of the vertebrae, the surgeon indicates the starting point for the drill and the desired end point (Figure 1a and 1b). At this point the surgeon can attach a set of planes to the central axis of the screw (Figure $2 \mathrm{a}$ and $2 \mathrm{~b}$ ). Each of these planes shows a slice through the CT data set. Thus the axis is used as a guidance path for a camera, whose view is shown in a separate window. By default, this camera is aimed at the slice perpendicular to the axis and it is moved along it by means of the thumbwheel located in the lower left corner of the window (Figure 2a). This view is especially useful to assess the correct positioning of the screw within the narrow isthmus. The camera can also be switched to a view containing the screw. This is used to check the correct upward angle of the inserted screw (Figure 2b). Once the screw is inserted, its angulation can still be adjusted and its dimensions adapted to obtain the optimal fixation of the vertebrae (Figure 3a). This procedure is then repeated for the contralateral screw. The design process thus consists of a loop where 2D and $3 \mathrm{D}$ are alternatively or simultaneously checked and adjusted.

\subsection{Personalized Drill Guide}

In order to enhance the precision and the safety of the screw insertion, a mechanical drill guide is derived from the planned trajectories of the screws (Figure $3 \mathrm{~b}$ ). The aim is to have a patient dependent drill guide that exactly fits to the posterior part of vertebra $\mathrm{C} 2$, incorporating drill holes indicating the correct position and orientation for the screws $[16,17]$. The reason the drill guide does not fit on 


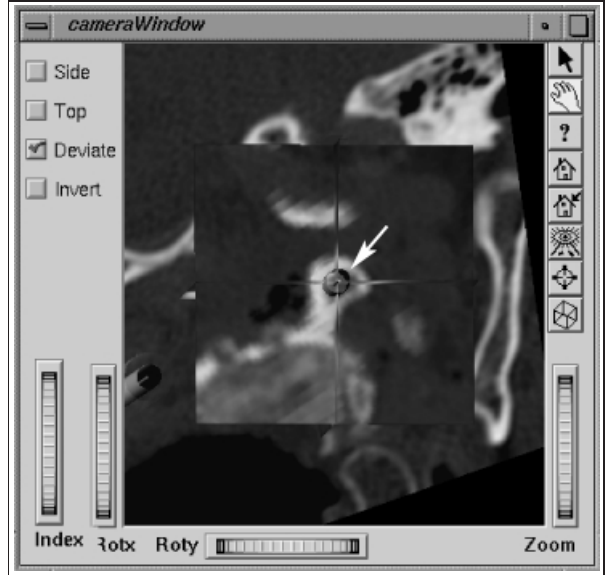

(a)

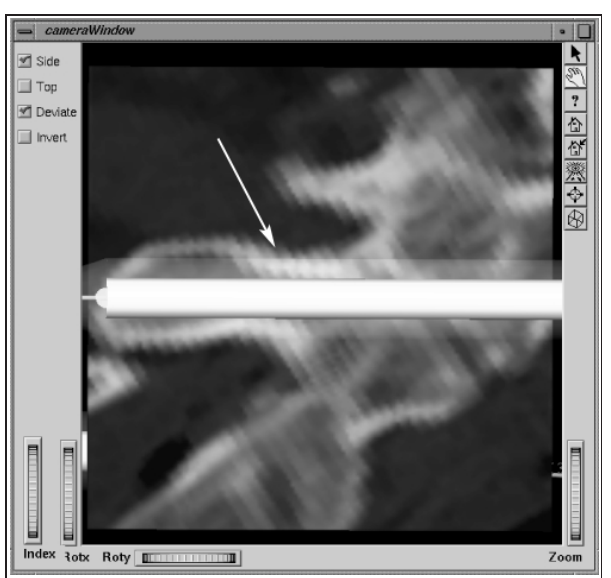

(b)

Fig. 2. (a) Reslice through the CT data, orthogonal to the inserted screw with arrow indicating the screw transversing the isthmus of C2 (b) Reslice through the CT data, containing the screw with arrow indicating the level at which Figure 1a was resliced

vertebra $\mathrm{C} 1$, is the possible change in relation between $\mathrm{C} 1$ and $\mathrm{C} 2$. They do not behave as one rigid body and so their relative position might be different at the time of CT scan and at the time of actual surgery. The drill guide can only be placed on the posterior part of $\mathrm{C} 2$ in one unique way. A CAD/CAM program uses the shape of the vertebra and the $3 \mathrm{D}$ information of the planned drill paths to design the drill guide. Shape and trajectories are available from the planning. The drill guide then is produced by stereolithography. We have opted not to use fully computer assisted spine surgery [18,19,20] because of the three major advantages a mechanical drill guide has to offer. First of all, only a low investment is required since no expensive computer equipment and 3D optical tracking hardware is needed in the operating room. Furthermore, the drill guide does not significantly increase the complexity of the surgery. And lastly, no preoperative referencing is needed, since the position of the drill guide upon the posterior part of the vertebra is unique. Of course there is a downside to this approach. At the time of operation the trajectories are fully fixed, no deviation from the planning is possible in the operating theater. Computer assisted surgery is much more flexible and allows continuous adjustment of the planning.

\section{Validation}

Five cadavers were CT scanned. Based on the planning, personalized drill guides were made. After insertion using these drill guides, postoperative CT scans were acquired under the same imaging conditions as for the preoperative images. The screws are made of $\mathrm{Ti}$, so they generate very little metallic streak artifact. The 


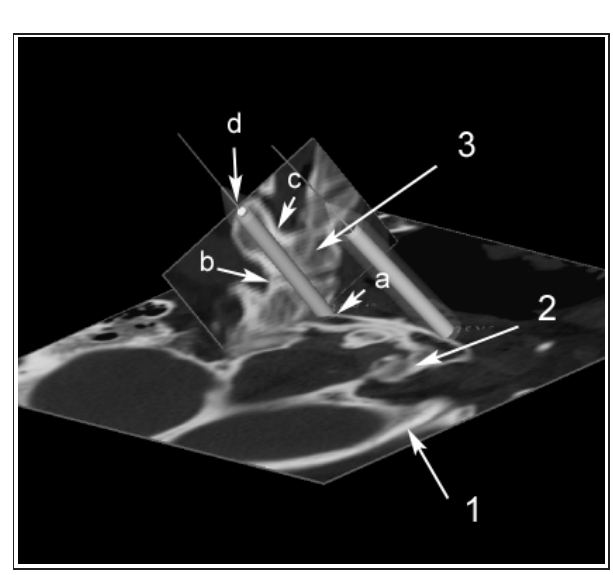

(a)

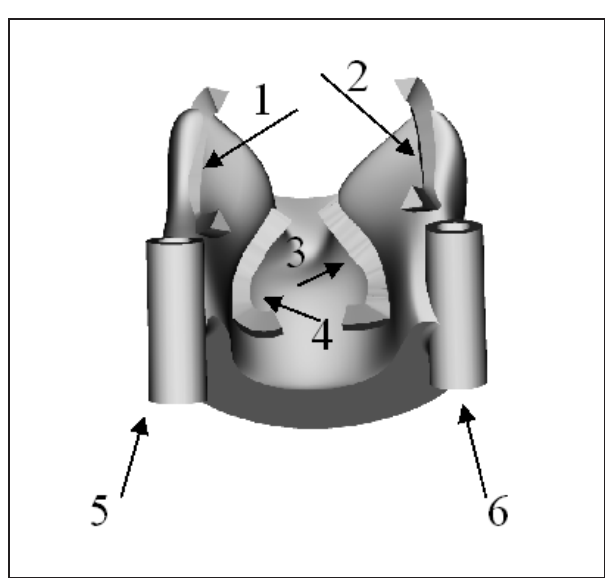

(b)

Fig. 3. (a) Single viewpoint containing one transverse slice, two planned screws and a reslice through one of the axes; $1=$ skull base, $2=$ vertebra $\mathrm{C} 1,3=$ vertebra $\mathrm{C} 2 ; \mathrm{a}=$ origin of the screw, $\mathrm{b}=$ joint between $\mathrm{C} 1$ and $\mathrm{C} 2$ that has to be stabilized, $\mathrm{c}=$ smallest diameter through which the screw needs to travel, $\mathrm{d}=$ end point of the screw (b) Full frontal view of the personalized mechanical drill guide. 1 to 4: connection interfaces ("knife-edges") between the drill guide and the vertebra; 5 and 6 : right and left drill hole

postoperative images therefore show very little distortion. In order to be able to view the planned screw superposed correctly upon the inserted one, the pre- and postoperative images have to be registered. This is achieved by using a technique, previously described [21]. Visualizing the planning on the result already leads to a qualitative assessment of the insertion (Figure $4 \mathrm{a}$ and $4 \mathrm{~b}$ ). To get quantitative results the following procedure was devised. In the postoperative images both screws were segmented by thresholding. This results in a voxel cloud of which the center of gravity is found. The principal axis through this center is found by analyzing eigenvalues and eigenvectors associated with the voxel cloud. At this point we can mathematically compare the axis of the planned screw with the one derived from the inserted screw. Since we know only the coordinates of the origin and endpoint of the planned screws, it is evident we should only find the corresponding points in the inserted screws and compare the coordinates. In order to do this, we look up the $z$ coordinate of the planned screws and calculate in the same $z$ plane the $x$ and $y$ coordinates of the inserted screws. The planned coordinates are then subtracted from the calculated ones to obtain $\Delta x$ and $\Delta y$. The coordinate system is defined as follows. The $z$ axis runs caudocranial, the $y$ axis anteroposterior and the $x$ axis from left to right. Another way of comparing the plan and its execution, is to assess the angles between them when projected onto sagittal, coronal and transverse planes. In addition to that the angle between the two axis directions was computed. The results of the measurements 
can be found in Table 1. Finally we have asked the neurosurgeon to qualitatively judge each resultant insertion, by placing it into either of four categories: clinically good, clinically acceptable, clinically unacceptable and clinically bad.

\section{Results and Discussion}

Table 1 presents the comparison between the planned and the inserted screws. The first part of the table lists the differences between $x$ and $y$ coordinates of planned and inserted screws on the left and right hand side. Looking at the numbers, one cadaver clearly stands out. Indeed, cadaver number two seems to have extremely large values for $\Delta y$, especially with respect to the end points. However, this can be entirely attributed to human error. During the insertion of the screws into the C1-C2 complex of cadaver number 2, it was observed by the neurosurgeon that the personalized drill guide did not fit at all, contrary to the other cadavers. Since this problem was not encountered with one of the other cadavers, a mere switch of drill guides did not take place. The only plausible explanation for this fact, is that probably the wrong cadaver was set up for the operation. Its results thus were excluded from all computations, as they are not relevant. When we compare the mean of the absolute differences in Table 1 , it is obvious that $\Delta x$ on average is smaller than $\Delta y$. However a plausible explanation can be offered for this observation. When the drill guide is used, the direction of the drilling forces coincides with the y direction almost perfectly. This results in a displacement of the template in that direction. Looking at the standard deviations of the differences, one will observe that the standard deviations of $\Delta y$ are much larger than those of $\Delta x$. This is probably due to the fact that the tendency of the drill guide to shift is much larger in the $y$ direction.

The second part of Table 1 lists the different angular deviations that have been computed.It is obvious that the angular deviations in the sagittal and coronal plane on average are larger than in the transverse plane. Hence, we conclude that the shift not only occurs in the $y$ direction, but also in the $z$ direction (as seen in the sagittal projection). This would corroborate our assumption that the drill guide slides away from its optimal posterior position as a consequence of the drilling force being exerted. The qualitative assessment of the screw insertion yielded following results. Of the relevant insertions i.e. all except cadaver 2, two screws from a total of eight were judged to be clinically unacceptable. In both cases there was a deviation of the screw axis that can only be attributed to a displacement of the drill guide. However, in none of the cases the screw invaded the spinal canal, nor was there any evidence of vascular compromise. Both screws were rejected only because their trajectory deviated too far from the ideal one (Figure 4a). Three screws were clinically well placed, three were clinically acceptable. In cadaver number 1 both screws were very well placed. This can be seen in Figure 4b. 
Table 1. Comparison of planned screws versus inserted screws. The first part of the table lists the absolute differences (in $\mathrm{mm}$ ) between $(x, y)$ coordinates of origin and end point in planned and inserted screws. The second part of the table lists the angles (in degrees) between planned and inserted screws.

$\mathrm{L}=$ left, $\mathrm{R}=$ right; $\mu=$ mean, $\sigma=$ standard deviation; subscripts $o$ and $e$ denote origin and end point respectively.

\begin{tabular}{|c||c|c|c|c||c|c|c|c|}
\hline \multicolumn{1}{|c||}{} & \multicolumn{3}{c||}{ Absolute Differences } & \multicolumn{5}{c|}{ Angles } \\
\hline Cadaver & $\Delta x_{o}$ & $\Delta y_{o}$ & $\Delta x_{e}$ & $\Delta y_{e}$ & Total & Sagittal & Coronal & Transverse \\
\hline \hline 1L & 0.75 & 2.13 & 0.07 & 0.23 & 2.37 & 2.04 & 1.23 & 1.92 \\
\hline 1R & 1.36 & 2.85 & 0.30 & 1.17 & 4.26 & 3.79 & 2.55 & 2.90 \\
\hline 2L & 0.46 & 8.96 & 0.26 & 14.79 & 4.84 & 4.58 & 1.36 & 2.63 \\
\hline 2R & 0.07 & 1.62 & 2.23 & 11.75 & 9.03 & 8.47 & 5.35 & 3.86 \\
\hline 3L & 0.22 & 7.12 & 0.26 & 1.43 & 5.11 & 5.10 & 1.10 & 0.05 \\
\hline 3R & 1.36 & 7.16 & 1.10 & 5.75 & 5.93 & 5.91 & 0.78 & 0.58 \\
\hline 4L & 2.20 & 5.20 & 0.39 & 4.38 & 2.64 & 0.43 & 5.01 & 3.11 \\
\hline 4R & 2.83 & 2.12 & 1.29 & 2.30 & 2.04 & 0.01 & 4.11 & 2.32 \\
\hline 5L & 1.64 & 6.92 & 0.34 & 7.09 & 8.07 & 8.04 & 4.44 & 0.69 \\
\hline 5R & 1.84 & 3.48 & 0.01 & 4.93 & 7.17 & 6.92 & 4.73 & 1.99 \\
\hline \hline$\mu$ (ex. 2) & 1.52 & 4.62 & 0.47 & 3.41 & 4.70 & 4.03 & 2.99 & 1.70 \\
\hline$\sigma$ (ex. 2) & 0.81 & 2.24 & 0.47 & 2.47 & 2.27 & 2.98 & 1.78 & 1.13 \\
\hline
\end{tabular}

\section{Conclusion and Future Developments}

As was already stated [22] we can certainly conclude that the use of computer assistance may help in placing $\mathrm{C} 1-\mathrm{C} 2$ transarticular screws. It certainly can assist navigation within $\mathrm{C} 2$. However, as we have observed, the drill guide's positioning is not yet stable enough to cope with the forces exerted during drilling. This instability can lead to unacceptable deviations from the optimal screw placement as a slight error in direction may result in a significant error in the position of the tip of the screw. The next 3 cadavers that are scheduled for the operation will have the advantage of being treated with a slightly different drill guide. Having a better contact with the spinous process of $\mathrm{C} 2$, this one is designed to have greater stability in the direction of the drilling force. As for the surgeon, this preoperative planning system allows him/her to rehearse the screw insertion in a more life-like way than by studying only the 2D CT images. It requires a minor time investment on his/her part, but this increases significantly the level of safety and tranquility associated with the procedure.

\section{Acknowledgments}

The work discussed here belongs, partly to the EU-funded Stereotactic LaserNeurosurgery project (nr. BMH4-CT96-0716), and partly to the EU-funded Brite Euram III PISA project (nr. BRPR CT97 0378). Partners in the latter are Materialise NV, Belgium; Philips Medical Systems BV, the Netherlands: ICS-AD; DePuy International Ltd, UK; Ceka NV, Belgium; K.U. Leuven, Belgium: ESAT/Radiology \& Div. Biomechanics; University of Leeds, UK: Research School of Medicine. 


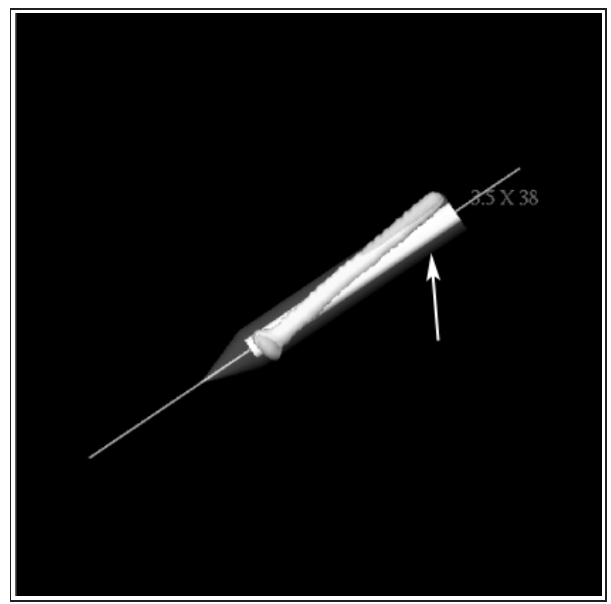

(a)

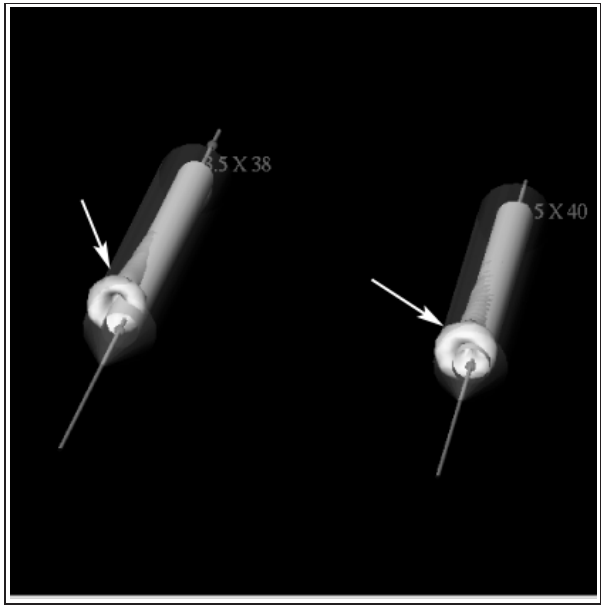

(b)

Fig. 4. (a) Sagittal view of the right hand screw in cadaver 5 superposed upon the planned screw. The arrow indicates the displacement error that prompted the rejection of this insertion. (b) View of both screws in cadaver 1 superposed upon the planned screws. They were judged to be very well placed. The arrows indicate the very small deviation which exists between the inserted and the planned screws.

\section{References}

1. Magerl F, Seeman PS. Stable Posterior Fusion of the Atlas and Axis by Transarticular Screw Fixation. In Kehr P. Weidner A (eds). Cervical Spine. Vienna, Springer Verlag 322 - 317, 1987860

2. Grob D, Jeanneret B, Aebi M, et al. Atlanto-axial Fusion with Transarticular Screw Fixation. J. Bone Joint Surg [Br] 1991; 73: 972 - 976 860, 861

3. Stillerman CB, Wilson JA. Atlanto-axial Stabilization with Posterior Transarticular Screw Fixation: Technical Description and Report of 22 Cases. Neurosurgery 1993; 32: 948 - 955 860, 861

4. Panjabi CG, Dvorak J, Duranceau J, et al. Three-dimensional Movements of the Upper Spine. Spine 1988; 13: 726 - 730861

5. Wilke HJ, Fischer K, Kuger A, Magerl F, Claes L, Wörsdörfer O. In Vitro Investigations of Internal Fixation Systems of the Upper Cervical Spine. II: Stability of Posterior Atlanto-axial Fixation Techniques. Eur Spine J 1992; 1: 191 - 199861

6. Gallie WE. Fractures and Dislocations of the Upper Cervical Spine. Am J Surg 1939; 46: 495 - 499861

7. Brooks AL, Jenkins EW. Atlanto-axial Arthrodesis by the Wedge Compression Method. J Bone Joint Surg [Am] 1978; 60: 279 - 284861

8. Grob D, Dvorak J, Panjabi M. Posterior Occipitocervial Fusion. A Preliminary Report of a New Technique. Spine 1991; 16(Suppl): S17 - S20

9. Madawi AA, Casey ATH, Solanki GA, Tuite G, VEres R, Crockard HA. Radiological and Anatomical Evaluation of the Atlantoaxial Transarticular Screw Fixation Technique. J Neurosurg 1997; 86: 961 - 968861 
10. Sasso RC, Jeanneret B, Fischer K. Occipitocervical Fusion with Posterior Plate and Screw Instrumentation. A Long-term Follow-up Study. Spine 1994; 19: 2364 2368861

11. Jeanneret B, Magerl F. Primary Posterior Fusion C1-2 in Odontoid Fractures: Indications, Technique and Results of Transarticular Screw Fixation. J Spinal Disord 1992; 5: $464-475861$

12. Apfelbaum RI. Screw Fixation of the Upper Cervical Spine: Indications and Techniques. Contemp Neurosurg 1994; 16: 1 - 8 861

13. Smith MD, Phillips WA, Hensinger RN. Complications of Fusion to the Upper Cervical Spine. Spine 1991; 16: 702 - 705861

14. Coric D, Branch CL Jr, Wilson JA, Robinson JC. Arteriovenous Fistula as a Complication of C1-C2 Transarticular Screw Fixation: Case Report and Review of the Literature. J Neurosurg 1996; 85: 340 - 343861

15. Verstreken K, Van Cleynenbreugel J, Martens K, Marchal G, van Steenberghe D, Suetens P. An Image-Guided Planning System for Endosseous Oral Implants. IEEE Transactions on Medical Imaging 1998; 17: 842 - 852861

16. Van Brussel K, Vander Sloten J, Van Audekercke R, Swaelens B, Vanden Berghe L, Fabry G. A Medical Image Based Template for Pedicle Screw Insertion. Conference Proceedings CARS'98, volume II: 347 - 354862

17. Goffin J, Van Brussel K, Vander Sloten J, Van Audekercke R, Smet M-H, Marchal G, Van Craen W, Swaelens B, Verstreken K. 3D-CT Based, Personalized Drill Guide for Posterior Transarticular Screw Fixation at C1-C2: Technical Note. Neuro-Orthopedics 1999; 25: 47 - 56862

18. Merloz P, Tonetti J, et al. Computer Assisted Spine Surgery. Clinical Orthopaedics and Related Research 1997; 337: 86 - 96863

19. Schwarzenbach O, Berlemann U, et al. Accuracy of Computer-Assisted Pedicle Screw Placement. An In Vivo Computed Tomography Analysis. Spine 1997; 22: $452-458 \quad 863$

20. Berlemann U, Monin D, Arm E, Nolte L-P, Ozdoba C. Planning and Insertion of Pedicle Screws with Computer Assistance. Journal of Spinal Disorders 1997; 10: $117-124863$

21. Maes F, Collignon A, Vandermeulen D, Marchal G, Suetens P. Multimodality image registration by maximization of mutual information, IEEE Transactions on Medical Imaging 1997; 16: 187-198 864

22. Gebhard JS, Schimmer RC, Jeanneret B. Safety and Accuracy of Transarticular Screw Fixation C1-C2 Using an Aiming Device. An Anatomic Study. Spine 1998; 23: $2185-2189866$ 